\title{
Direct electron imaging of dislocation activities in nanocrystalline molybdenum nanopillars
}

Haw-Wen Hsiao ${ }^{1}$ and Jian-Min Zuo ${ }^{2}$

${ }^{1}$ University of Illinois Urbana-Champaign, Urbana, Illinois, United States, ${ }^{2}$ University of Illinois at Urbana-Champaign, Urbana, Illinois, United States

Nanocrystalline materials are widely used for their special properties, including structural applications with high strength. The strength enhancement comes from the inhibited dislocation activities by the reduced grain size following the Hall-Petch relationship [1]. However, fundamental understanding of the strengthening mechanism(s) is still lacking. Transmission electron microscopy (TEM) with its high spatial and temporal resolution is an ideal tool to address this, but there are significant challenges for its application. Here, we utilize in-situ electron imaging to monitor dislocation activities and structural evolution in nanocrystalline molybdenum (Mo) nanopillars. In contrast to face-centered cubic (FCC) metals, body-centered cubic (BCC) ones show distinctive size dependent mechanical behaviors upon the grain refinement. One reason is that dislocations in the BCC crystals exhibit a large difference in mobility between the screw and edge characters, with the strength and plasticity controlled by the screw dislocation motions. A consequence in the fine-grained BCC metallic systems is their reduced strain rate sensitivity (SRS) [2]. Multiple mechanisms were proposed to explain the reduced SRS in nanocrystalline BCC metals, including the change in the dominant dislocation character [3], the diminished difference in dislocation mobility [4], the enhanced interactions with grain boundaries [5], and etc. Such divergence reflects the complexity of dislocation dynamics at small scales.

The fine microstructure in nanocrystalline materials is a major challenge in dislocation characterization. We have developed a novel approach based the combination of in-situ observation of dislocation motions and nanostructure determination using scanning electron nanodiffraction [6]. Figure 1 shows the experimental approach. Nanopillars were fabricated from a nanocrystalline Mo film by the focus ion beam method (FIB). A highly sensitivepicoindenter was used to conduct compression testing on the nanopillars and simultaneously record the mechanical response from the material. By recording the image video during deformation, we can monitor dislocation activities using the activity intensity method as described in Ref [7]. As demonstrated in Fig. 1C, the activities in microstructure (red) are strongly correlated with the mechanical behavior (black). Before compression, SEND was performed on the nanopillars in a FEI Titan Themis Z scanning transmission electron microscope. A $~ 1 \mathrm{~nm}$-sized electron probe was scanned over a 2D grid of real space probe positions with a step size of $5 \mathrm{~nm}$. The diffraction pattern at each position was successively captured using a CMOS camera. The local structure information from the diffraction dataset help identify the contrast change in the recorded electron images, extracting more details in the microstructure during deformation.

Using the described approach above, we have tested nanopillars of 200 to $300 \mathrm{~nm}$ in diameters. Our findings demonstrate that grain boundaries (GBs) play an essential role that contribute to the strength and plasticity. In the early stage of deformation, the strain is mainly accommodated by dislocation interactions and GB deformation, while most of activities are strongly confined by GBs. In the later stage, intermittent transgranular dislocation avalanches dominate the plasticity. Dislocation with edge and screw characters are both observed, and their motions are restricted by GBs. Together, the results provide critical insights into strengthening via grain refinement in a refractory metal. 
A
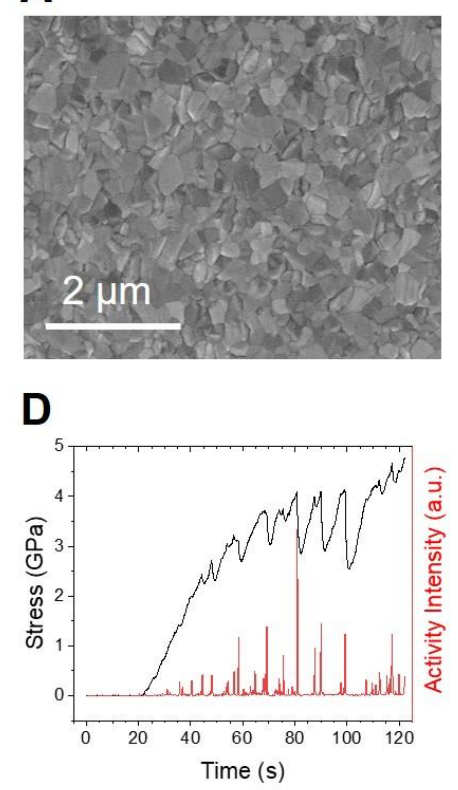

B
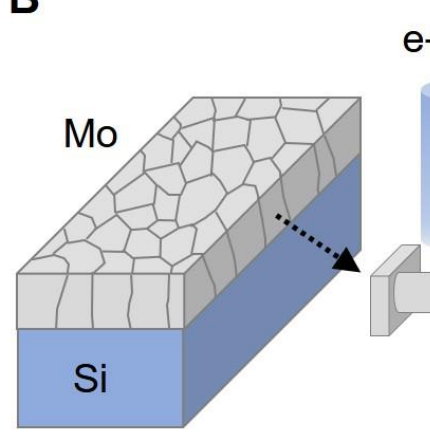

e-beam

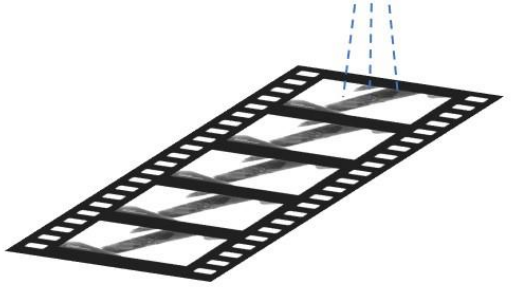

C

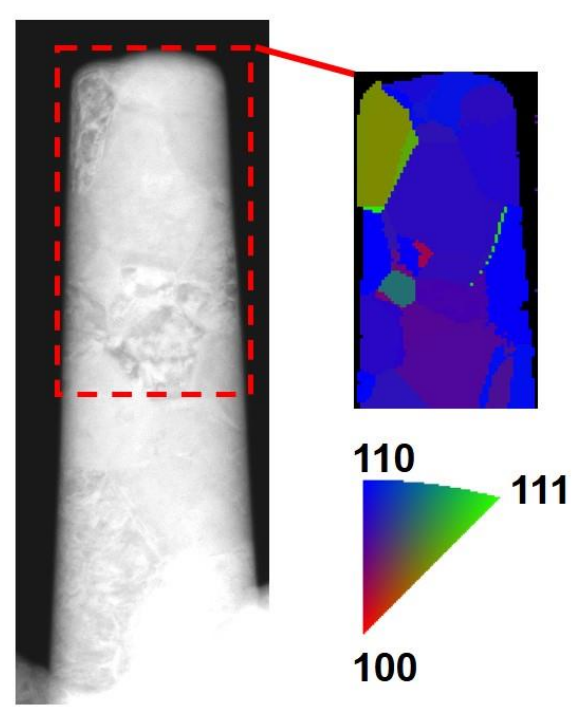

Figure 1. Fig. 1 (A) A plane-view SEM image of a nanocrystalline Mo thin film used for nanopillar fabrication. (B) Experimental set-up of an in-situ compression testing combining with ex-situ SEND on a nanopillar. (C) A STEM image of a nanopillar and its orientation map measured by SEND. (D) The measured stress-time curve (black) is plotted with the intensity curve (red) of activities in microstructure during deformation.

\section{References}

1. Meyers, M.A., A. Mishra, and D.J. Benson, Mechanical properties of nanocrystalline materials. Progress in Materials Science, 2006. 51(4): p. 427-556.

2. Wei, Q., et al., Effect of nanocrystalline and ultrafine grain sizes on the strain rate sensitivity and activation volume: $f c c$ versus bcc metals. Materials Science and Engineering: A, 2004. 381(1): p. 71-79.

3. Cheng, G.M., et al., Grain Size Effect on Deformation Mechanisms of Nanocrystalline bcc Metals. Materials Research Letters, 2013. 1(1): p. 26-31.

4. Huang, R., et al., Flow Stress in Submicron BCC Iron Single Crystals: Sample-size-dependent Strainrate Sensitivity and Rate-dependent Size Strengthening. Materials Research Letters, 2015. 3(3): p. 121127.

5. Schneider, A.S., et al., Correlation between Critical Temperature and Strength of Small-Scale bcc Pillars. Physical Review Letters, 2009. 103(10): p. 105501.

6. Zuo, J.-M., Electron Nanodiffraction, in Springer Handbook of Microscopy, P.W. Hawkes and J.C.H. Spence, Editors. 2019, Springer International Publishing: Cham. p. 905-969.

7. Hsiao, H.-W., et al., Shear banding mechanism in compressed nanocrystalline ceramic nanopillars. Physical Review Materials, 2019. 3(8): p. 083601. 